\title{
Glycated haemoglobin, plasma glucose and diabetic retinopathy: cross-sectional and prospective analyses
}

\author{
Q.Z. Liu ${ }^{1}$, D.J.Pettitt ${ }^{1}$, R.L.Hanson ${ }^{1}$, M.A.Charles ${ }^{1}$, R.Klein ${ }^{2}$, P.H. Bennett ${ }^{1}$ and W.C. Knowler $^{1}$ \\ ${ }^{1}$ Diabetes and Arthritis Epidemiology Section, Phoenix Epidemiology and Clinical Research Branch, National Institute of Diabetes \\ and Digestive and Kidney Diseases, Phoenix, Arizona, USA \\ ${ }^{2}$ Department of Ophthalmology, University of Wisconsin Medical School, Madison, Wisconsin, USA
}

\begin{abstract}
Summary. Among Pima Indians with Type 2 (non-insulindependent) diabetes mellitus the relationships between glycated haemoglobin $\left(\mathrm{HbA}_{1}\right)$, fasting or 2-h post-load plasma glucose and diabetic retinopathy were examined by crosssectional and prospective analyses, and the strengths of the associations were directly compared by receiver operating characteristic analysis. In the cross-sectional analysis, $\mathrm{HbA}_{1}$, fasting and 2-h plasma glucose were each significantly related to retinopathy among 789 diabetic subjects by separate logistic models. In a stepwise multiple logistic model in which $\mathrm{HbA}_{1}$, fasting and 2-h plasma glucose were included, $\mathrm{HbA}_{1}$ was selected as having the strongest association with retinopathy and neither fasting nor 2-h plasma glucose contributed significantly to the model once $\mathrm{HbA}_{1}$ was entered. Similarly, in the prospective analysis, $\mathrm{HbA}_{1}$, fasting and 2 -h plasma glucose all predicted retinopathy in 227 diabetic subjects by separate proportional-hazards models. In a stepwise proportional-hazards model with $\mathrm{HbA}_{1}$, fasting and 2-h plasma glucose available to the model, $\mathrm{HbA}_{1}$ was again selected as having the strongest association with the incidence of retino-
\end{abstract}

pathy, and neither fasting nor 2-h plasma glucose significantly added to the prediction of retinopathy. A receiver operating characteristic analysis was used to determine if $\mathrm{HbA}_{1}$ was statistically significantly better than fasting or 2 -h plasma glucose in assessing the risk for retinopathy. In neither the crosssectional nor the prospective data did the area under the receiver operating characteristic curve for $\mathrm{HbA}_{1}$ differ significantly from that for fasting or 2-h plasma glucose ( $p>0.05$ for each). In conclusion, $\mathrm{HbA}_{1}$, an integrated measure of blood glucose concentration over a period of $2-3$ months, is slightly more closely associated with the prevalence and incidence of diabetic retinopathy than a single blood glucose determination. However, the differences between $\mathrm{HbA}_{1}$ and fasting or 2-h plasma glucose in assessing the association with or the risk for retinopathy are not significant.

Key words: Glycated haemoglobin $A_{1}$, plasma glucose, diabetic retinopathy, risk factor, receiver operating characteristic (ROC) analysis, Pima Indians.
Glycated haemoglobin measurement is a method for estimating the degree of hyperglycaemia over a period of 2-3 months. On the other hand, plasma glucose concentrations vary with recent activity levels, metabolic stress, food intake, and time of day [1-3]. It is therefore of interest to see whether subjects with high glycated haemoglobin are at greater risk for diabetic retinopathy and, if so, whether glycated haemoglobin can provide a better measure of risk for retinopathy than a single blood glucose determination. Previous studies have shown that higher glycated haemoglobin [4-7] and higher plasma glucose [8-14] indicate an increased risk of diabetic retinopathy. However, so far, it is not known whether glycated haemoglobin is a better tool for investigation of risk for diabetic retinopathy than a single blood glucose determination.

In the present study, glycated haemoglobin $\mathrm{A}_{1}\left(\mathrm{HbA}_{1}\right)$ and plasma glucose were evaluated as risk factors for diabetic retinopathy by cross-sectional and prospective analyses, and the strengths of the associations of $\mathrm{HbA}_{1}$ and plasma glucose with diabetic retinopathy were compared using receiver operating characteristic (ROC) analysis.

\section{Subjects, materials and methods}

\section{Subjects and measurements}

A longitudinal study of diabetes and its complications has been conducted among the Pima Indian residents of the Gila River Indian Community of Arizona since 1965 [15]. This population has a very high prevalence and incidence of Type 2 (non-insulin-dependent) diabetes mellitus. Approximately every 2 years, the residents of the community who are 5 years old or older have, regardless of health, been asked to participate in a standardised medical examination 
Table 1. "Retinopathy levels" graded by a modification of Airile House Classification Scheme modified from references [19,20]

\section{Retinopathy Description}

level

\begin{tabular}{|c|c|}
\hline Level 10 & No retinopathy. \\
\hline Level 11 & $\begin{array}{l}\text { Questionable diabetic retinopathy, usually one que- } \\
\text { stionable microaneurysm. }\end{array}$ \\
\hline Level 12 & $\begin{array}{l}\text { Retinopathy that is non-diabetic in nature but could } \\
\text { be mistaken for diabetic retinopathy. }\end{array}$ \\
\hline Level 14 & $\begin{array}{l}\text { Definite hard exudates, soft exudates or intraretinal } \\
\text { microvascular abnormalities in the absence of mi- } \\
\text { croaneurysms. }\end{array}$ \\
\hline
\end{tabular}

Level 15 Retinal haemorrhage without microaneurysms.

Level 20 Microaneurysms only without other diabetic lesions.

Level 31 Microaneurysms and one or more of the following: retinal haemorrhage, but total of haemorrhages and microaneurysms less than those in standard photograph 2 A [19], hard exudates, soft exudates questionably present, intraretinal microvascular abnormalities questionably present, venous beading questionably present, and venous loops definitely present.

Level 41 Microaneurysms and one or more of the following: soft exudates definitely present, and intraretinal microvascular abnomalities definitely present but less than that necessary for level 51 .

Level 51 Microaneurysms and one or more of the following: venous beading definitely present, haemorrhages and microaneurysms equalling or exceeding those in standard photograph $2 \mathrm{~A}$ [19], and intraretinal microvascular abnormalities present and equalling or exceeding those in standard photograph $8 \mathrm{~A}$ [19] in 2 fields.

Level $60+{ }^{a}$ Any of several levels of severity of proliferative retinopathy, including neovascularization, fibrous proliferation, vitreous haemorrhage and preretinal haemorrhage, scars of panretinal photocoagulation, and/or retinopathy ungradable because of vitreous haemorrhage obscuring the retina, phthisis bulbi, or enucleation secondary to a complication of diabetic retinopathy.

${ }^{a}$ Level $60+$ including levels $60,65,70,80$

Table 2. Crude associations between $H b A_{1}$, plasma glucose and prevalence or incidence of diabetic retinopathy

\begin{tabular}{|c|c|c|}
\hline \multirow[t]{2}{*}{ Variables } & $\begin{array}{l}\text { Cross-sectional } \\
\text { analyses }\end{array}$ & $\begin{array}{l}\text { Prospective } \\
\text { analyses }\end{array}$ \\
\hline & Odds ratio & Incidence rate ratio \\
\hline \multicolumn{3}{|l|}{ Separate models ${ }^{\mathfrak{c}}$} \\
\hline Fasting plasma glucose & $3.47(2.29-5.24)$ & $4.31(1.97-9.43)$ \\
\hline 2-h plasma glucose & $3.82(2.51-5.82)$ & $5.72(2.31-14.16)$ \\
\hline $\mathrm{HbA}_{1}$ & $4.64(3.01-7.15)$ & $5.84(2.53-13.49)$ \\
\hline
\end{tabular}

${ }^{a}$ Odds ratio $(95 \% \mathrm{Cl})$ for each variable computed from a logistic regression model is shown comparing the 90 th with the 10 th percentiles.

Incidence rate ratio $(95 \% \mathrm{Cl}$ ) for each variable computed from a proportional-hazards model is shown comparing the 90th with the 10 th percentiles.

${ }^{\text {c}}$ Fasting, 2-h plasma glucose or $\mathrm{HbA}_{1}$ was analysed in each separate model with same number of subjects $(n=789$, cases of retinopathy $=271$ for cross-sectional analysis; $n=227$, new cases of retinopathy $=42$ for prospective analysis) which includes a medical history, a physical examination and laboratory tests. A 75-g oral glucose tolerance test (OGTT) was performed. Plasma glucose was measured by the ferricyanide method with an Autoanalyser (Technicon Instruments Corporation, Tarrytown, NY, USA). Diabetes was diagnosed by the criteria of the World Health Organisation [16], i.e. 2-h post-load plasma glucose concentration $\geq 11.1 \mathrm{mmol} / \mathrm{l}(\geq 200 \mathrm{mg} / \mathrm{dl})$. $\mathrm{HbA}_{1}$ was measured by agar gei electrophoresis $[17,18]$.

Since 1982, photographs of two standard fields for each eye have been taken, after pupillary dilatation, with a Canon CR4-45 NM non-mydriatic fundus camera in subjects aged 15 years or older. To determine retinopathy status, all fundus photographs were graded by trained readers who had no knowledge of the clinical data, in a masked fashion, using a modification of the Airlie House Classification Scheme that specified 13 levels of retinopathy for each eye [19, 20]. These retinopathy levels are shown in Table 1 . Retinopathy level 88 which could not be graded due to poor photo quality was not included in this table. The retinopathy level for a participant was derived from the most severely involved eye, or from one eye if only one could be graded. Retinopathy was defined by the presence of any microaneurysms, haemorrhages, exudates, or proliferative retinopathy (retinopathy level $\geq 14$ ).

The present study employs both cross-sectional and prospective analyses. In the cross-sectional analysis, data from the last examination of 789 diabetic subjects ( 296 men and 493 women) with both $\mathrm{HbA}_{1}$ and fundus photo data were used. In the prospective analysis, 227 diabetic subjects ( 61 men and 166 women), free of retinopathy at their first diabetic examination at which $\mathrm{HbA}_{1}$ was measured, were followed-up.

\section{Statistical analysis}

Cross-sectional analysis: prevalence rates of retinopathy were computed according to tertiles of $\mathrm{HbA}_{1}$, fasting and $2-\mathrm{h}$ plasma glucose. A logistic regression model was used to determine the associations of $\mathrm{HbA}_{1}$, fasting or 2-h plasma glucose with diabetic retinopathy. A stepwise multiple logistic regression model, which included $\mathrm{HbA}_{1}$, fasting and 2-h post-load plasma glucose, was used to select which of these variables was most closely associated with retinopathy.

Prospective analysis: 227 diabetic subjects without retinopathy were followed-up from the first diabetic examination at which $\mathrm{HbA}_{1}$ was measured. Follow-up lasted until development of retinopathy as detected at a subsequent examination or until the last examination. Five-year cumulative incidence rates were estimated according to tertiles of $\mathrm{HbA}_{1}$, fasting and 2-h plasma glucose by Kaplan-Meier survival curves. A proportional-hazards model was used to evaluate the associations between $\mathrm{HbA}_{1}$, fasting or 2 -h plasma glucose and incidence of diabetic retinopathy. A stepwise proportional-hazards analysis was used to identify the best predictor of retinopathy among the variables, $\mathrm{HbA}_{1}$, fasting and 2-h post-load plasma glucose. Variables measured at the beginning of follow-up for each subject were used in these analyses.

$\mathrm{HbA}_{1}$ and plasma glucose concentrations were approximately log - normally distributed, so the natural logarithms of these variables were used in logistic regression and proportional - hazards analyses. Odds ratios (with $95 \%$ confidence intervals, CI) and incidence rate ratios (with $95 \% \mathrm{CI}$ ) are shown comparing the 90 th with the 10 th percentiles of fasting or $2-\mathrm{h}$ plasma glucose or $\mathrm{HbA}_{1}$.

ROC analysis: since logistic regression and proportional hazards analyses do not allow for the direct comparison of correlated measures, this was accomplished by comparing the area under ROC curves. The ROC curve represents the diagnostic properties of a test by plotting sensitivity as a function of 1 -specificity. In the cross-sectional analysis, the area under the ROC curve represents the probability that the test will correctly distinguish between a subject chosen at random from among those who have retinopathy and one chosen from among those who do not have it [21]. In the prospective 

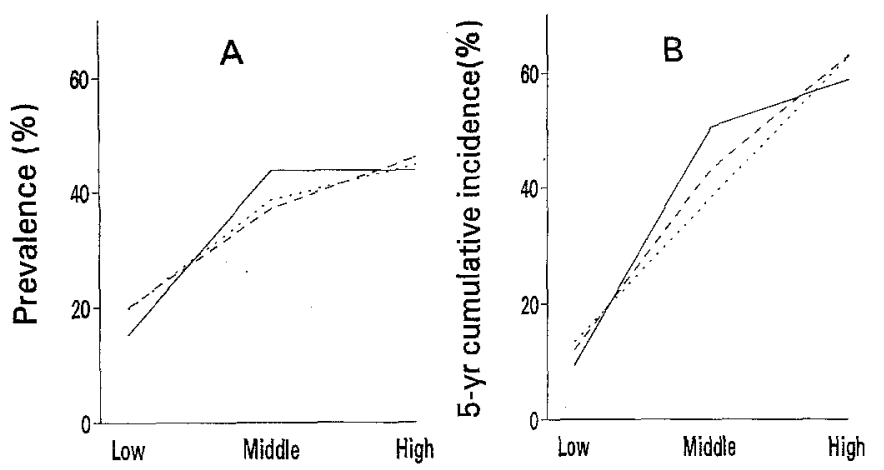

Fig. 1. (A) Prevalence of diabetic retinopathy according to tertiles of $\mathrm{HbA}_{\mathrm{t}}(-)$ fasting $(\cdots)$ and 2 -h plasma glucose (---). (B) Fiveyear cumulative incidence of retinopathy according to tertiles of $\mathrm{HbA}_{1}(-)$, fasting $(.$.$) ) and 2$-h plasma glucose (-.--)

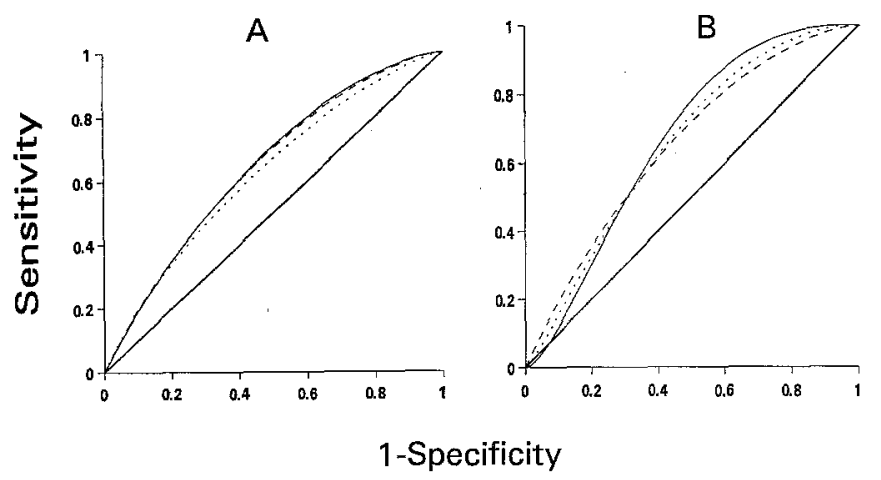

Fig.2(A,B). The comparisons of the sensitivity and specificity of $\mathrm{HbA}_{1}(-)$, fasting $(\cdots)$ ) and 2 -h plasma glucose (---), as risk factors for diabetic retinopathy, by receiver operating characteristic (ROC) analyses based on the cross-sectional (A) and the prospective data (B) (The thick black line represents the chance diagonal)

analysis, it represents the probability of correctly identifying which subject will develop retinopathy by the end of follow-up: In the present study, the areas under the ROC curves for $\mathrm{HbA}_{1}$, fasting or 2-h plasma glucose were computed with the LABROC program and the significance of the difference between areas under ROC curves was calculated using the CLABROC program [22].

\section{Results}

\section{Cross-sectional analysis}

Of 789 diabetic subjects, 271 (34.3\%) had retinopathy. Of these 271 subjects, $254(93.7 \%)$ had background retinopathy (levels 14-51 in Table 1); and 17 (6.3\%) had proliferative retinopathy (level $60+$ ).

Figure $1 \mathrm{~A}$ shows the prevalence rates of diabetic retinopathy by tertiles of $\mathrm{HbA}_{1}$, fasting and 2-h plasma glucose. Table 2 shows the crude association of diabetic retinopathy with $\mathrm{HbA}_{1}$, fasting or 2-h plasma glucose. Each was significantly related to diabetic retinopathy. In a stepwise multiple logistic regression analysis in which $\mathrm{HbA}_{1}$, fasting and 2 -h plasma glucose were available to the model, $\mathrm{HbA}_{1}$ was selected as having the strongest association with retinopathy and neither fasting nor 2 -h plasma glucose concentrations contributed significantly to the
Q.Z. Liu et al.: Glycated haemoglobin, plasma glucose and retinopathy

model after $\mathrm{HbA}_{1}$ had entered (not shown). Since age, sex, and duration of diabetes might confound the above association, further analyses were performed to control for these variables by logistic regression. In separate logistic models for fasting and 2-h plasma glucose and $\mathrm{HbA}_{1}$, each of these variables, when controlled for age, sex and duration of diabetes, was significantly associated with diabetic retinopathy $(p<0.01$ for each, the model with $\mathrm{HbA}_{1}$ also included the interaction term of $\mathrm{HbA}_{1}$ with duration of diabetes). In a stepwise multiple logistic model (with age, sex, duration of diabetes, $\mathrm{Hb} \mathrm{A}_{1}$ and fasting and 2-h plasma glucose available in the model) $\mathrm{HbA}_{1}$ $(p<0.01)$ was again selected as having a strong association with retinopathy, second only to duration, and neither fasting nor 2-h plasma glucose concentration contributed significantly to the model.

\section{Prospective analysis}

Of 227 diabetic subjects without retinopathy at baseline, $42(18.5 \%)$ subsequently developed retinopathy during 1.4-6.0 (mean 3.3) years of follow-up. Of 42 subjects with new retinopathy, all had background retinopathy (levels 14-51 in Table 1), and none had proliferative retinopathy.

Figure $1 \mathrm{~B}$ shows the 5-year cumulative incidence of retinopathy according to tertiles of $\mathrm{HbA}_{1}$, fasting and $2-\mathrm{h}$ plasma glucose. Table 2 shows the crude association of diabetic retinopathy with $\mathrm{HbA}_{1}$, fasting or 2-h plasma glucose, each of which predicted diabetic retinopathy. In a stepwise proportional-hazards model in which $\mathrm{HbA}_{1}$, fasting and 2-h plasma glucose were available to the model, $\mathrm{HbA}_{1}$ was selected as having the strongest predictive value and neither fasting nor 2 -h plasma glucose significantly added to the prediction of diabetic retinopathy (not shown). Since duration of diabetes, age and sex might confound these results, further analyses were performed to control for these variables by the proportional-hazards model. In separate proportional-hazards models for fasting and 2-h plasma glucose and $\mathrm{HbA}_{1}$, each of these variables, when controlled for age, sex and duration of diabetes, predicted retinopathy ( $p<0.01$ for each). In a stepwise proportional-hazards model (with age, sex, duration of diabetes, fasting and 2-h plasma glucose and $\mathrm{HbA}_{1}$ available in the model) $\mathrm{HbA}_{1}(p<0.01)$ was again selected as having the strongest association with the incidence of retinopathy, and neither fasting nor 2-h plasma glucose concentration significantly added to the prediction of diabetic retinopathy. Duration was also selected $(p<0.05)$.

\section{ROC analysis}

ROC curves for the association of retinopathy with $\mathrm{HbA}_{1}$, fasting and 2-h plasma glucose are shown in Figure $2 \mathrm{~A}$ (cross-sectional data) and 2B (prospective data). The area under a curve represents the degree of association between the measure of glycaemia $\left(\mathrm{HbA}_{1}\right.$, fasting or 2-h plasma glucose) and retinopathy. The greater the area, the greater the probability of correctly identifying those who 
have, or will develop, retinopathy. The area under the diagonal chance line ( 0.5$)$ represents the probability of correctly distinguishing, by chance alone, between two subjects, one with and one without retinopathy. The fact that all curves lie above the chance line reflects the finding that all three variables were associated with retinopathy. The curves also show that the three tests have similar sensitivities at thresholds of equal specificity. In the cross-sectional analysis, the area under the ROC curve for $\mathrm{HbA}_{1}$ (area \pm standard error (SE), $0.644 \pm 0.040$ ) was not significantly different from that for fasting $(0.623 \pm 0.020)$ or for 2-h plasma glucose $(0.642 \pm 0.020, p>0.05$ for each comparison). Similarly, in the prospective atalysis, the area under the ROC curve for $\mathrm{HbA}_{1}(0.663 \pm 0.040)$ was not significantly different from that for fasting $(0.652 \pm 0.042)$ or for 2 -h plasma glucose $(0.650 \pm 0.044$, $p>0.05$ for each comparison)

\section{Discussion}

In the present study of subjects with Type 2 (non-insulindependent) diabetes, $\mathrm{HbA}_{1}$ was significantly associated with the prevalence and incidence of diabetic retinopathy. Similar results have been reported in other studies [4-7]. Fasting and 2-h plasma glucose were also significantly related to the prevalence and the incidence of diabetic retinopathy in the present study. Many studies suggest a relationship between plasma glucose and retinopathy [8-14], but others are less convincing $(5,23)$. The inconsistency of these results may lie in the limitations of a single plasma glucose determination which may be influenced by age, activity level, metabolic stress, food intake, and time of day [1]. By contrast, $\mathrm{HbA}_{1}$ has proven to be an acceptable method for determining the degree of hyperglycaemia because it provides an integrated measure of blood glucose level over the previous $2-3$ months and is not affected by time of day, recent activity, food intake and other factors [1-3].

Our results indicated that $\mathrm{HbA}_{1}$, fasting and 2-h plasma glucose concentrations were similar in their associations with both the prevalence and the incidence of diabetic retinopathy. As indicated in the longitudinal analyses by odds ratios for the comparison of the 90 th with the 10 th percentiles, which range from 4.3 to 5.8 , each measure of glycaemia was a good predictor of the subsequent development of retinopathy. However, in stepwise multiple regression models in which $\mathrm{HbA}_{1}$, fasting and 2-h plasma glucose were available to the models, $\mathrm{HbA}_{1}$ was selected as having the strongest association with retinopathy in cross-sectional and prospective analyses. Similarly, Klein et al. [4] found that $\mathrm{HbA}_{1}$ was a stronger predictor of diabetic retinopathy than a single plasma glucose determination by logistic regression. Since logistic regression and proportional-hazards analyses do not allow for the direct comparison of correlated measures, these methods do not determine whether $\mathrm{HbA}_{1}$ is significantly superior to plasma glucose in assessing the risk for retinopathy.

However, ROC analysis, a direct comparison of the two methods, as shown in the present paper, may provide a better estimate of the relative strengths of these associ- ations [21]. ROC analyses indicated no significant differences between $\mathrm{HbA}_{1}$ and fasting or 2-h plasma glucose in assessing the association with or the risk for retinopathy in our cross-sectional and prospective data. This suggested that, in this population, any one of these three measures was adequate in association or prediction models. Given any one, addition of the other two made no statistically significant contribution. However, the correlation between measures of glycaemia in predicting retinopathy may be influenced by the magnitude of the day to day variability in the plasma glucose. In subjects with more day to day variability, $\mathrm{HbA}_{1}$ and plasma glucose concentrations would be expected to be less correlated, and the relative abilities of $\mathrm{HbA}_{1}$ and plasma glucose concentrations to predict retinopathy might be different from the present findings.

In conclusion, $\mathrm{HbA}_{1}$ is slightly more closely associated with the prevalence and incidence of diabetic retinopathy, and the logistic regression and proportional hazards analysis assign all the significance to the one variable. However, ROC analysis demonstrates that there is not a significant difference between $\mathrm{HbA}_{1}$ and fasting or 2-h plasma glucose in assessing the risk for retinopathy in either the cross-sectional or the prospective data. Nevertheless, $\mathrm{HbA}_{1}$ may have practical advantages in assessing the risk for retinopathy in diabetic patients, especially for clinical and epidemiologic purposes, because of logistic difficulties in administering an OGTT to patients or requiring patients to be examined after an overnight fast. According to these results, the choice of measurement of glycaemia for exploring relationships to retinopathy can be determined by practical rather than scientific considerations.

Acknowledgements. The authors thank the members of the Gila River Indian Community for their co-operation in this investigation; the staff of the Diabetes and Arthritis Epidemiology Section, NIDDK, for conducting the studies; and the graders at the University of Wisconsin Fundus Photograph Reading Center.

\section{References}

1. Little RR, England JD, Weidmeyer H et al. (1988) Relationship of glycosylated hemoglobin to oral glucose tolerance: implications for diabetes screening. Diabetes 37: 60-64

2. Compagnucci P, Cartechini MG, Bolli G, Feo PD, Santeusanio F, Brunetti P (1981) The importance of determining irreversibly glycosylated hemoglobin in diabetics. Diabetes 30:607 612

3. Bunn HF (1981) Evaluation of glycosylated hemoglobin in diabetic patients. Diabetes 30: 613-617

4. Klein R, Klein BEK, Moss SE, Davis MD, DeMets DL (1988) Glycosylated hemoglobin predicts the incidence and progression of diabetic retinopathy. JAMA 260: 2864-2871

5. Janka HU, Warram JH, Rand LI, Krolewski A (1989) Risk factors for progression of background retinopathy in long-standing IDDM. Diabetes 38: 460-464

6. Kalter-Leibovici O, Dyk DJV, Leibovici L et al. (1991) Risk factors for development of diabetic nephropathy and retinopathy in Jewish IDDM patients. Diabetes 40: 204-210

7. Nathan DM, Singer DE, Godine JE, Harrington CH, Perlemuter LC (1986) Retinopathy in older type II diabetes: association with glucose control. Diabetes 35: 797-801

8. Teuscher A, Schnell H, Wilson PWF (1988) Incidence of diabetic retinopathy and relationship to baseline plasma glucose and blood pressure. Diabetes Care 11:246-251 
9. Knowler WC, Bennett PH, Ballintine EJ (1980) Increased incidence of retinopathy in diabetics with elevated blood pressure: a six-year follow-up study in Pima Indians. N Engl J Med 302: 645-650

10. Pettitt DJ, Knowler WC, Lisse JR, Bennett PH (1980) Development of retinopathy and proteinuria in relation to plasma glucose concentrations in Pima Indians. Lancet II: 1050-1052

11. West KM, Erdreich LJ, Stober JA (1980) A detailed study of risk factors for retinopathy and nephropathy in diabetes. Diabetes 29: 501-508

12. Dorf A, Ballintine EJ, Bennett PH, Miller M (1976) Retinopathy in Pima Indians: relationships to glucose level, duration of diabetes, age at diagnosis of diabetes, and age at examination in a population with a high prevalence of diabetes mellitus. Diabetes 225: 554-560

13. Krolewski AS, Warram JH, Rand LI, Christlieb AR, Busick EJ, Kahn CR (1986) Risk of proliferative diabetic retinopathy in juvenile-onset type I diabetes: a 40-yr follow-up study. Diabetes Care 9: 443-452

14. Ballard DJ, Melton LJ III, Dwyer MS et al. (1986) Risk factors for diabetic retinopathy: a population-based study in Rochester, Minnesota. Diabetes Care 9: 334-342

15. Bennett PH, Burch TA, Miller M (1971) Diabetes mellitus in American (Pima) Indians. Lancet II: 125-128

16. Diabetes mellitus (1985) Report of a WHO Study Group. WHO Technical Report Series 727. Geneva, World Health Organization, pp 9-17

17. Menard L, Dempsey ME, Blankstein LA, Aleyassine H, Wacks M, Soeldner JS (1980) Quantitative determination of glycosylated hemoglobin A1 by agar gel electrophoresis. Clin Chem 26: 1598-1602
18. Hayes EJ, Gleason RE, Soeldner JS, Wacks M, Blankstein L (1981) Measurement of hemoglobin A1 by liquid chromatography and by agar gel electrophoresis compared. Clin Chem 27: $476-479$

19. Diabetic Retinopathy Study Research Group (1981) A modification of the Airlie House classification of diabetic retinopathy. Invest Ophthalmol Vis Sci 21:210-226

20. Klein R, Klein BEK, Magli YL et al. (1986) An alternative method of grading diabetic retinopathy. Ophthalmology 93: 1183-1187

21. Hanley JA, McNeil BJ (1982) The meaning and use of the area under a receiver operating characteristic (ROC) curve. Radiology 143:29-36

22. Metz CE, Wang PL, Kronman HB (1984) A new approach for testing the significance of differences between ROC curves measured from correlated data. In: Deconinck F (ed) Proceedings of the VIIIth Conference on Information Processing in Medical Imaging. M Nijoff, The Hague, pp 432-445

23. Nelson RG, Wolfe JA, Horton MB, Pettitt DJ, Bennett PH, Knowler WC (1989) Proliferative retinopathy in NIDDM: incidence and risk factors in Pima Indians. Diabetes 38: 435-440

Received: 6 October 1992 and in revised form: 21 December 1992

Dr. D.J.Pettitt

Diabetes and Arthritis Epidemiology Section NIDDK

1550 East Indian School Road

Phoenix, Arizona 85014

USA 